\title{
THE SOLUTION OF STOCHASTIC TIME-DEPENDENT FIRST ORDER DELAY DIFFERENTIAL EQUATIONS USING BLOCK SIMPSON'S METHODS
}

\author{
B. O. OSU ${ }^{1,3}$, C. CHIBUISI ${ }^{2}$, G. A. EGBE $^{3} \&$ V. C. EGENKONYE ${ }^{1}$ \\ ${ }^{1}$ Department of Mathematics, Michael Okpara University of Agriculture, Umudike, Nigeria \\ ${ }^{2}$ Department of Insurance, University of Jos, Jos, Nigeria \\ ${ }^{3}$ Department of Mathematics Abia State University Uturu, Nigeria
}

\begin{abstract}
This paper deals with numerical solution of stochastic time-dependent delay differential equations (STDDDEs) using block Simpson's mechanism for pace number $k=2,3$ and 4 without intercalation approach in calculating the delay term containing random values. The multistep collocation method was used to derive the discrete schemes of the proposed method through matrix inversion technique of each step number. The convergence and region of absolute stability of these discrete schemes were examined. The implementation of these discrete schemes was worked-out in block forms to solve some stochastic time-dependent first order delay differential equations. It was observed that the scheme for step number $k=4$ performed better and faster in terms of accuracy than the schemes for step number $k=3$ and 2 respectively after the comparisons with their exact solutions and other existing methods. KEYWORDS: Stochastic delay differential equation, block method, linear multistep method. Mathematics Subject Classification 2010: 34K28; 65F30; 90C30; 90 C26
\end{abstract}

Received: Dec 31, 2020; Accepted: Jan 20 2021; Published: Feb 12, 2021; Paper Id.: IJMCARJUN20211

\section{INTRODUCTION}

In recent years, the study of numerical solution of stochastic delay differential equations has attracted the attention of many researchers. Previously, stochastic processes were used in solving ODE and have shown less advantage in obtaining accurate solutions. Stochastic delay differential equation (SDDE) is a stochastic differential equation where the increment of the process depends not only on current state but also on the history part of the system being modelled. The history part is called a delay term which contains the random values. The applications of SDDEs can be seen in many real life phenomena involving celestial and quantum mechanics, nuclear, theoretical and economic dynamics and control.

In this research work, we are concerned with using Simpson's Mechanism for pace number k= 2, 3 and 4 to obtain the numerical solutions of an autonomous d-dimensional Ito stochastic time-dependent delay differential equation (STDDDE) of the form as studied by [1]

$$
\begin{aligned}
& d X(z)=f(X(z), X(z-\tau), z) d z+g(X(z), X(z-\tau), z) d W(z) \text { for } z>z_{0}, \tau>0 \\
& X(z)=p(z), \text { for } z \leq z_{0}
\end{aligned}
$$

where $p(z)$ is the initial function, $X(z)$ is the stochastic process of the current state, $\tau$ is the lag, $(z-\tau)$ is the 
lag argument and $X(z-\tau)$ is the solution of the lag argument on the drift part and $X(z-\tau) d W(z)$ is the solution of the delay term containing the m-dimensional Brownian motion or Wiener process on the diffusion part of (1).The drift and diffusion coefficients $a, b^{j}: \mathfrak{R}^{d} \rightarrow \mathfrak{R}^{d}$ for $j=1, \ldots, m$ are Borel-measurable functions.

Most researchers applied Euler-Maruyama scheme to develop a continuous split-step scheme of SDDE on a continuous interval $t_{0} \leq t \leq t_{a}$ in order to obtain its numerical solutions with the application of intercalation techniques in appraising the lag argument but in this work, we used Block Simpson's Method as a linear multistep collocation method to discretize STDDDE in (1) on a discrete interval $\left[t_{0}, t_{a}\right)$ in order to derive its discrete schemes from the continuous formulations of each step number through matrix inversion method. These discrete schemes obtained were applied in solving some STDDDEs without the application of the intercalation method in evaluating the lag argument containing random values. The results obtained revealed the order and error constants, consistency, zero stability, convergence, absolute stability, efficiency and accuracy of the proposed method better than the other existing methods. Many researchers have successfully carried-out some research work using intercalation method in assessing the lag argument of SDDEs but the accuracy of the results obtained were not preserved. [2] proposed a new improved split-step backward Euler method for SDDEs with time-dependent delay where a piecewise linear interpolation is used to approximate the solution at the delayed points with restriction on the stepsize. The authors [3, 4] adopted the continuous-time model for stochastic volatility involving state-dependent delayed response and applied the Euler-Maruyama discrete-time approximation in the strong convergence sense to stimulate the noise applying interpolation techniques in computing the delay term.[5] considered the strong convergence and the mean-square stability of the split-step backward Euler method to linear SDDEs with constant lag which was evaluated with an interpolation technique.[6] used Euler-Maruyama scheme to illustrate some numerical examples of SDDEs and introduced interpolation techniques in investigating the lag term.[7] studied the convergence and stability of the numerical solution of stochastic state-dependent delay differential equations with Euler-Maruyama scheme to develop the continuous split-step scheme with the interpolation techniques been applied for the examination of the delay term containing random values.

[8], stated one of the difficulties some scholars always experienced in the use of these intercalation approach to assess the lag argument.

We applied ideas of the sequence formulated by [9] and discrete-time Gaussian white process whose mean $\mu$ is 0 and the standard deviation $\sigma$ is 1derived by [10] to overcome the difficulty faced in using the interpolation techniques for the evaluation of the delay and wiener terms. We inserted the investigated terms into the stochastic time-dependent first order delay differential equations before obtaining its numerical solutions. Then we applied block Simpson's method to solve some stochastic time-dependent delay differential equations having the assessed lag and wiener terms. To determine the importance of the proposed method, we compared with the already existing methods investigated by [6,7] at constant pence size $S$.

On the other hands, authors [11,12,13 and 14] have successfully worked on the approximate solutions of the first order DDEs without the use of intercalation approach in evaluating the lag term. One of the differences between SDDEs and DDEs is that the delay term of the SDDEs contains random values while that of the DDEs does not. The proposed method above shall also be compared with some of the numerical results of DDEs carried out by $[11,14]$ to ascertain its supremacy over the other existing methods. 


\section{CONSTRUCTION TECHNIQUES}

\subsection{Construction of Multistep Collocation Method}

In [15], a $k$-step multistep collocation method with $d$ collocation points was obtained as

$$
y(x)=\sum_{r=0}^{n-1} \alpha_{r}(x) y_{n+r}+s \sum_{r=0}^{d-1} \beta_{r}(x) f(x, y(x))
$$

where $\alpha_{r}(x)$ and $\beta_{r}(x)$ are continuous coefficients of the method defined as

$$
\begin{aligned}
& \alpha_{r}(x)=\sum_{w=0}^{n+d-1} \alpha_{r, w+1} x^{w} \text { for } r=\{0,1, \ldots, n-1\}(3) \\
& s \beta_{r}(x)=\sum_{w=0}^{n+d-1} s \beta_{r, w+1} x^{w} \text { for } r=\{0,1, \ldots, d-1\}
\end{aligned}
$$

where $X_{0}, \ldots, X_{d-1}$ are the $d$ collocation points and $x_{a+r}, r=0,1,2, \ldots, n-1$ are the $n$ arbitrarily chosen interpolation points.

To get $\alpha_{r}(x)$ and $\beta_{r}(x),[16]$ arrived at a matrix equation of the form

$$
B A=\mathrm{I}
$$

where $I$ is the elementary matrix of dimension is $(n+d) \times(n+d)$ while $B$ and $A$ are matrices defined as

$$
\begin{aligned}
B & =\left[\begin{array}{ccccccc}
\alpha_{0,1} & \alpha_{1,1} & \cdots & \alpha_{n-1,1} & s \beta_{0,1} & \cdots & s \beta_{d-1,1} \\
\alpha_{0,2} & \alpha_{1,2} & \cdots & \alpha_{n-1,2} & s \beta_{0,2} & \cdots & s \beta_{d-1,2} \\
\vdots & \vdots & \vdots & \vdots & \vdots & \vdots & \vdots \\
\alpha_{0, n+d} & \alpha_{1, n+d} & \cdots & \alpha_{n-1, n+d} & s \beta_{0, n+d} & \cdots & s \beta_{d-1, n+d}
\end{array}\right] \\
A & =\left[\begin{array}{ccccc}
1 & x_{a} & x_{a}^{2} & \cdots & x_{a}^{n+d-1} \\
1 & x_{a+1} & x_{a+1}^{2} & \cdots & x_{a+1}^{n+d-1} \\
\vdots & \vdots & \vdots & \vdots & \vdots \\
1 & x_{a+n-1} & x_{a+n-1}^{2} & \cdots & x_{a+n-1}^{n+d-1} \\
0 & 1 & 2 x_{0} & \cdots & (n+d-1) x_{0}^{n+d-2} \\
\vdots & \vdots & \vdots & \vdots & \vdots \\
0 & 1 & 2 x_{d-1} & \cdots & (n+d-1) x_{d-1}^{n+d-2}
\end{array}\right]
\end{aligned}
$$

From the equation (5), $B=A^{-1}$ gives the continuous coefficients of the continuous scheme (2).

Subsequently, the formulations of the continuous form of the BSMs for pace numbers $\mathrm{k}=2,3$ and 4 shall be obtained. 
2.2 Construction of Block Simpson's Methods (BSMs) for $k=2$

Here, $n=1$ and $d=3$.Then, (2) becomes

$$
y(x)=\alpha_{0}(x) y_{a}+s \beta_{0}(x) f_{a}+s \beta_{1}(x) f_{a+1}+s \beta_{2}(x) f_{a+2}
$$

The matrix $A$ in (5) becomes

$$
A=\left(\begin{array}{cccc}
1 & x_{a} & x_{a}^{2} & x_{a}^{3} \\
0 & 1 & 2 x_{a} & 3 x_{a}^{2} \\
0 & 1 & 2 s+2 x_{a} & 3\left(s+x_{a}\right)^{2} \\
0 & 1 & 4 s+2 x_{a} & 3\left(2 s+x_{a}\right)^{2}
\end{array}\right)
$$

Using Maple 18, the matrix inverse $B=A^{-1}$ from which the continuous scheme is obtained shall be evaluated and simplified at $x=x_{a+1}$ and $x=x_{a+2}$ to obtain

$$
\begin{aligned}
& y_{a+1}=y_{a}+\frac{5}{12} s f_{a}+\frac{2}{3} s f_{a+1}-\frac{1}{12} s f_{a+2} \\
& y_{a+2}=y_{a}+\frac{1}{3} s f_{a}+\frac{4}{3} s f_{a+1}+\frac{1}{3} s f_{a+2}
\end{aligned}
$$

\subsection{Construction of Block Simpson's Methods (BSMs) for $k=3$}

In this case, $n=1$ and $d=4$. Hence, (2) takes the expression

$$
y(x)=\alpha_{0}(x) y_{a}+s \beta_{0}(x) f_{a}+s \beta_{1}(x) f_{a+1}+s \beta_{2}(x) f_{a+2}+s \beta_{3}(x) f_{a+3}
$$

The matrix $A$ in (5) becomes

$$
A=\left(\begin{array}{ccccc}
1 & x_{a} & x_{a}^{2} & x_{a}^{3} & x_{a}^{4} \\
0 & 1 & 2 x_{a} & 3 x_{a}^{2} & 4 x_{a}^{3} \\
0 & 1 & 2 s+2 x_{z} & 3\left(s+x_{a}\right)^{2} & 4\left(s+x_{a}\right)^{3} \\
0 & 1 & 4 s+2 x_{a} & 3\left(2 s+x_{a}\right)^{2} & 4\left(2 s+x_{a}\right)^{3} \\
0 & 1 & 6 s+2 x_{a} & 3\left(3 s+x_{a}\right)^{2} & 4\left(3 s+x_{a}\right)^{3}
\end{array}\right)
$$

Using Maple 18, the matrix inverse $B=A^{-1}$ from which the continuous scheme is obtained shall be evaluated and simplified at $x=x_{a+1}, x=x_{a+2}$ and $x=x_{a+3}$ to derive

$$
\begin{aligned}
& y_{a+1}=y_{a}+\frac{3}{8} s f_{a}+\frac{19}{24} s f_{a+1}-\frac{5}{24} s f_{a+2}+\frac{1}{24} s f_{a+3} \\
& y_{a+2}=y_{a}+\frac{1}{3} s f_{a}+\frac{4}{3} s f_{a+1}+\frac{1}{3} s f_{a+2}
\end{aligned}
$$




$$
y_{a+3}=y_{a}+\frac{3}{8} s f_{a}+\frac{9}{8} s f_{a+1}+\frac{9}{8} s f_{a+2}+\frac{3}{8} s f_{a+3}
$$

\subsection{Construction of Block Simpson's Methods (BSMs) for $k=4$}

Let $n=1$ and $d=5$. Hence, (2) takes the form

$$
y(x)=\alpha_{0}(x) y_{a}+s \beta_{0}(x) f_{a}+s \beta_{1}(x) f_{a+1}+s \beta_{2}(x) f_{a+2}+s \beta_{3}(x) f_{a+3}+s \beta_{4}(x) f_{a+4}
$$

Also the matrix $A$ in (5) becomes

$$
A=\left(\begin{array}{cccccc}
1 & x_{a} & x_{a}^{2} & x_{a}^{3} & x_{a}^{4} & x_{a}^{5} \\
0 & 1 & 2 x_{a} & 3 x_{a}^{2} & 4 x_{a}^{3} & 5 x_{a}^{4} \\
0 & 1 & 2 s+2 x_{a} & 3\left(s+x_{a}\right)^{2} & 4\left(s+x_{a}\right)^{3} & 5\left(s+x_{a}\right)^{4} \\
0 & 1 & 4 s+2 x_{a} & 3\left(2 s+x_{a}\right)^{2} & 4\left(2 s+x_{a}\right)^{3} & 5\left(2 s+x_{a}\right)^{4} \\
0 & 1 & 6 s+2 x_{a} & 3\left(3 s+x_{a}\right)^{2} & 4\left(3 s+x_{a}\right)^{3} & 5\left(3 s+x_{a}\right)^{4} \\
0 & 1 & 8 s+2 x_{a} & 3\left(4 s+x_{a}\right)^{2} & 4\left(4 s+x_{a}\right)^{3} & 5\left(4 s+x_{a}\right)^{4}
\end{array}\right)
$$

Applying Maple 18, the matrix inverse $B=A^{-1}$ from which the continuous scheme is obtained shall be evaluated and simplified at $x=x_{a+1}, x=x_{a+2}, x=x_{a+3}$ and $x=x_{a+4}$ to derive

$$
\begin{aligned}
& y_{a+1}=y_{a}+\frac{251}{720} s f_{a}+\frac{323}{360} s f_{a+1}-\frac{11}{30} s f_{a+2}+\frac{53}{360} s f_{a+3}-\frac{19}{720} s f_{a+4} \\
& y_{a+2}=y_{a}+\frac{29}{90} s f_{a}+\frac{62}{45} s f_{a+1}+\frac{4}{15} s f_{a+2}+\frac{2}{45} s f_{a+3}-\frac{1}{90} s f_{a+4} \\
& y_{a+3}=y_{a}+\frac{27}{80} s f_{a}+\frac{51}{40} s f_{a+1}+\frac{9}{10} s f_{a+2}+\frac{21}{40} s f_{a+3}-\frac{3}{80} s f_{a+4} \\
& y_{a+4}=y_{a}+\frac{14}{45} s f_{a}+\frac{64}{45} s f_{a+1}+\frac{8}{15} s f_{a+2}+\frac{64}{45} s f_{a+3}+\frac{14}{45} s f_{a+4}
\end{aligned}
$$

\section{ANALYSIS OF CONVERGENCE}

Here, the analysis of order, error constant, consistency, zero stability and region of absolute stability of (10), (13) and (16) are tested.

\subsection{Order and Error Constant}

For (10), the order and error constant are obtained as follows

$$
\begin{aligned}
& c_{0}=c_{1}=c_{2}=c_{3}=\left(\begin{array}{ll}
0 & 0
\end{array}\right)^{T} \\
& c_{4}=\left(\begin{array}{ll}
\frac{1}{24} & 0
\end{array}\right)^{T}
\end{aligned}
$$


Therefore, (10) has an order, $q=3$ and error constants, $\left(\begin{array}{cc}\frac{1}{24} & 0\end{array}\right)^{T}$

By using the same approach for (13) and can be presented as follows

$$
\begin{aligned}
& c_{0}=C_{1}=C_{2}=C_{3}=C_{4}=\left(\begin{array}{lll}
0 & 0 & 0
\end{array}\right)^{T} \\
& c_{5}=\left(\begin{array}{lll}
-\frac{19}{720} & -\frac{1}{90} & -\frac{3}{80}
\end{array}\right)^{T}
\end{aligned}
$$

Therefore, (13) has order $q=4$ and error constants, $\left(\begin{array}{ccc}-\frac{19}{720} & -\frac{1}{90} & -\frac{3}{80}\end{array}\right)^{T}$

With the same approach, (16) and can be presented as

$$
\begin{aligned}
& \mathcal{C}_{0}=C_{1}=C_{2}=C_{3}=C_{4}=C_{5}=\left(\begin{array}{llll}
0 & 0 & 0 & 0
\end{array}\right)^{T} \\
& c_{6}=\left(\begin{array}{llll}
\frac{3}{160} & \frac{1}{90} & \frac{3}{160} & 0
\end{array}\right)^{T}
\end{aligned}
$$

From the expression, (16) has order $q=5$ and error constants, $\left(\frac{3}{160}, \frac{1}{90}, \frac{3}{160}, 0\right)^{T}$

\subsection{Consistency}

In [17], the local truncation error (LTE) is stated as $\left\|E_{\psi}\right\|=C_{z+1} S^{z+1}+O\left(s^{z+2}\right)$ and for BSM (2) to be consistent, the order q must be greater than or equal to 1 . Let the LTE of this method BSM (2) be $C_{z+1} \neq 0$ as $z \geq 1$.

From section 3.0 above, the $C_{z+1} \neq 0$ for (10) is generated and shown as $C_{4}=\left(\frac{1}{24}, 0\right)^{Q}$ with order $z=(4,4)^{Q}$.

With the same process for (13), $C_{z+1} \neq 0$ for (13) is $C_{5}=\left(-\frac{19}{720},-\frac{1}{90},-\frac{3}{80}\right)^{Q}$ with order $z=(5,5,5)^{Q}$.

Using the same approach, (16), $C_{z+1} \neq 0$ for (16) is derived and shown as $\mathcal{C}_{6}=\left(\frac{3}{160}, \frac{1}{90}, \frac{3}{160}, 0\right)^{Q}$ with order $z=(6,6,6,6)^{Q}$ where $Q=T$

The BSM (2) is consistent having satisfied the above condition of order $q \geq 1$. 


\subsection{Zero Stability Analysis}

For (10), the zero stability is estimated as follows

$$
\left(\begin{array}{ll}
1 & 0 \\
0 & 1
\end{array}\right)\left(\begin{array}{l}
y_{a+1} \\
y_{a+2}
\end{array}\right)=\left(\begin{array}{ll}
0 & 1 \\
0 & 1
\end{array}\right)\left(\begin{array}{c}
y_{a-1} \\
y_{a}
\end{array}\right)+s\left(\begin{array}{cc}
\frac{2}{3} & -\frac{1}{12} \\
\frac{4}{3} & \frac{1}{3}
\end{array}\right)\left(\begin{array}{l}
f_{a+1} \\
f_{a+2}
\end{array}\right)+s\left(\begin{array}{cc}
0 & \frac{5}{12} \\
0 & \frac{1}{3}
\end{array}\right)\left(\begin{array}{c}
f_{a-1} \\
f_{a}
\end{array}\right)
$$

where

$$
H_{2}^{(1)}=\left(\begin{array}{ll}
1 & 0 \\
0 & 1
\end{array}\right), H_{1}^{(1)}=\left(\begin{array}{ll}
0 & 1 \\
0 & 1
\end{array}\right), J_{2}^{(1)}=\left(\begin{array}{cc}
\frac{2}{3} & -\frac{1}{12} \\
\frac{4}{3} & \frac{1}{3}
\end{array}\right) \text { and } L_{2}^{(1)}=\left(\begin{array}{cc}
0 & \frac{5}{12} \\
0 & \frac{1}{3}
\end{array}\right)
$$

The first characteristic polynomial is stated as

$$
\begin{gathered}
m(\varpi)=\operatorname{det}\left(\varpi H_{2}^{(1)}-H_{1}^{(1)}\right) \\
=\left|\varpi H_{2}^{(1)}-H_{1}^{(1)}\right|=0
\end{gathered}
$$

Now we have,

$$
\begin{aligned}
& m(\varpi)=\left|\varpi\left(\begin{array}{ll}
1 & 0 \\
0 & 1
\end{array}\right)-\left(\begin{array}{ll}
0 & 1 \\
0 & 1
\end{array}\right)\right|=\left|\left(\begin{array}{cc}
\varpi & 0 \\
0 & \varpi
\end{array}\right)-\left(\begin{array}{ll}
0 & 1 \\
0 & 1
\end{array}\right)\right| \\
& \Rightarrow m(\varpi)=\left(\begin{array}{cc}
\varpi & -1 \\
0 & \varpi-1
\end{array}\right)
\end{aligned}
$$

Using Maple (18) software, we obtain

$$
\begin{aligned}
& m(\varpi)=\varpi(\varpi-1) \\
& \Rightarrow \varpi(\varpi-1)=0 \\
& \Rightarrow \varpi_{1}=1, \varpi_{2}=0 . \text { Since }\left|\varpi_{i}\right| \leq 1, i=1,2, \text { the discrete schemes in (10) is zero stable. }
\end{aligned}
$$

Applying the same approach for (13), we have

$$
\left(\begin{array}{lll}
1 & 0 & 0 \\
0 & 1 & 0 \\
0 & 0 & 1
\end{array}\right)\left(\begin{array}{l}
y_{a+1} \\
y_{a+2} \\
y_{a+3}
\end{array}\right)=\left(\begin{array}{lll}
0 & 0 & 1 \\
0 & 0 & 1 \\
0 & 0 & 1
\end{array}\right)\left(\begin{array}{c}
y_{a-2} \\
y_{a-1} \\
y_{a}
\end{array}\right)+s\left(\begin{array}{ccc}
\frac{19}{24} & -\frac{5}{24} & \frac{1}{24} \\
\frac{4}{3} & \frac{1}{3} & 0 \\
\frac{9}{8} & \frac{9}{8} & \frac{3}{8}
\end{array}\right)\left(\begin{array}{l}
f_{a+1} \\
f_{a+2} \\
f_{a+3}
\end{array}\right)+s\left(\begin{array}{ccc}
0 & 0 & \frac{3}{8} \\
0 & 0 & \frac{1}{3} \\
0 & 0 & \frac{3}{8}
\end{array}\right)\left(\begin{array}{l}
f_{a-2} \\
f_{a-1} \\
f_{a}
\end{array}\right)
$$


where

$$
H_{2}^{(2)}=\left(\begin{array}{lll}
1 & 0 & 0 \\
0 & 1 & 0 \\
0 & 0 & 1
\end{array}\right), H_{1}^{(2)}=\left(\begin{array}{lll}
0 & 0 & 1 \\
0 & 0 & 1 \\
0 & 0 & 1
\end{array}\right), J_{2}^{(2)}=\left(\begin{array}{ccc}
\frac{19}{24} & -\frac{5}{24} & \frac{1}{24} \\
\frac{4}{3} & \frac{1}{3} & 0 \\
\frac{9}{8} & \frac{9}{8} & \frac{3}{8}
\end{array}\right) \text { and } L_{2}^{(2)}=\left(\begin{array}{ccc}
0 & 0 & \frac{3}{8} \\
0 & 0 & \frac{1}{3} \\
0 & 0 & \frac{3}{8}
\end{array}\right)
$$

Let the first characteristic polynomial be presented as

$$
\begin{gathered}
m(\varpi)=\operatorname{det}\left(\varpi H_{2}^{(2)}-H_{1}^{(2)}\right) \\
=\left|\varpi H_{2}^{(2)}-H_{1}^{(2)}\right|=0
\end{gathered}
$$

Now we have,

$$
\begin{aligned}
& m(\varpi)=\left|\varpi\left(\begin{array}{lll}
1 & 0 & 0 \\
0 & 1 & 0 \\
0 & 0 & 1
\end{array}\right)-\left(\begin{array}{lll}
0 & 0 & 1 \\
0 & 0 & 1 \\
0 & 0 & 1
\end{array}\right)\right|=\left|\left(\begin{array}{ccc}
\varpi & 0 & 0 \\
0 & \varpi & 0 \\
0 & 0 & \varpi
\end{array}\right)-\left(\begin{array}{lll}
0 & 0 & 1 \\
0 & 0 & 1 \\
0 & 0 & 1
\end{array}\right)\right| \\
& \Rightarrow m(\varpi)=\left(\begin{array}{ccc}
\varpi & 0 & -1 \\
0 & \varpi & -1 \\
0 & 0 & \varpi-1
\end{array}\right)
\end{aligned}
$$

The following are obtained using Maple (18) software,

$$
\begin{aligned}
& m(\varpi)=\varpi^{2}(\varpi-1) \\
& \Rightarrow \varpi^{2}(\varpi-1)=0 \\
& \Rightarrow \varpi_{1}=1, \varpi_{2}=0, \varpi_{3}=0
\end{aligned}
$$

.Since $\left|\varpi_{i}\right| \leq 1, i=1,2,3$, the discrete schemes in (13) is zero stable.

By the same technique (16) can be presented as follows

$$
\left(\begin{array}{llll}
1 & 0 & 0 & 0 \\
0 & 1 & 0 & 0 \\
0 & 0 & 1 & 0 \\
0 & 0 & 0 & 1
\end{array}\right)\left(\begin{array}{l}
y_{a+1} \\
y_{a+2} \\
y_{a+3} \\
y_{a+4}
\end{array}\right)=\left(\begin{array}{llll}
0 & 0 & 0 & 1 \\
0 & 0 & 0 & 1 \\
0 & 0 & 0 & 1 \\
0 & 0 & 0 & 1
\end{array}\right)\left(\begin{array}{c}
y_{a-3} \\
y_{a-2} \\
y_{a-1} \\
y_{a}
\end{array}\right)
$$




$$
+s\left(\begin{array}{cccc}
\frac{323}{360} & -\frac{11}{30} & \frac{53}{360} & -\frac{19}{720} \\
\frac{62}{45} & \frac{4}{15} & \frac{2}{45} & -\frac{1}{90} \\
\frac{51}{40} & \frac{9}{10} & \frac{21}{40} & -\frac{3}{80} \\
\frac{64}{45} & \frac{8}{15} & \frac{64}{45} & \frac{14}{45}
\end{array}\right)\left(\begin{array}{l}
f_{a+1} \\
f_{a+2} \\
f_{a+3} \\
f_{a+4}
\end{array}\right)+s\left(\begin{array}{cccc}
0 & 0 & 0 & \frac{251}{720} \\
0 & 0 & 0 & \frac{29}{90} \\
0 & 0 & 0 & \frac{27}{80} \\
0 & 0 & 0 & \frac{14}{45}
\end{array}\right)\left(\begin{array}{c}
f_{a-3} \\
f_{a-2} \\
f_{a-1} \\
f_{a}
\end{array}\right)
$$

where

$$
H_{2}^{(3)}=\left(\begin{array}{llll}
1 & 0 & 0 & 0 \\
0 & 1 & 0 & 0 \\
0 & 0 & 1 & 0 \\
0 & 0 & 0 & 1
\end{array}\right), H_{1}^{(3)}=\left(\begin{array}{llll}
0 & 0 & 0 & 1 \\
0 & 0 & 0 & 1 \\
0 & 0 & 0 & 1 \\
0 & 0 & 0 & 1
\end{array}\right), J_{2}^{(3)}=\left(\begin{array}{cccc}
\frac{323}{360} & -\frac{11}{30} & \frac{53}{360} & -\frac{19}{720} \\
\frac{62}{45} & \frac{4}{15} & \frac{2}{45} & -\frac{1}{90} \\
\frac{51}{40} & \frac{9}{10} & \frac{21}{40} & -\frac{3}{80} \\
\frac{64}{45} & \frac{8}{15} & \frac{64}{45} & \frac{14}{45}
\end{array}\right) \text { and } L_{1}^{(3)}=\left(\begin{array}{cccc}
0 & 0 & 0 & \frac{251}{720} \\
0 & 0 & 0 & \frac{29}{90} \\
0 & 0 & 0 & \frac{27}{80} \\
0 & 0 & 0 & \frac{14}{45}
\end{array}\right)
$$

In this case, the first characteristic polynomial is stated as

$$
\begin{gathered}
m(\varpi)=\operatorname{det}\left(\varpi H_{2}^{(3)}-H_{1}^{(3)}\right) \\
=\left|\varpi H_{2}^{(3)}-H_{1}^{(3)}\right|=0
\end{gathered}
$$

Now we have,

$$
\begin{aligned}
& m(\varpi)=\left|\left(\begin{array}{llll}
1 & 0 & 0 & 0 \\
0 & 1 & 0 & 0 \\
0 & 0 & 1 & 0 \\
0 & 0 & 0 & 1
\end{array}\right)-\left(\begin{array}{llll}
0 & 0 & 0 & 1 \\
0 & 0 & 0 & 1 \\
0 & 0 & 0 & 1 \\
0 & 0 & 0 & 1
\end{array}\right)\right|=\left|\left(\begin{array}{cccc}
\varpi & 0 & 0 & 0 \\
0 & \varpi & 0 & 0 \\
0 & 0 & \varpi & 0 \\
0 & 0 & 0 & \varpi
\end{array}\right)-\left(\begin{array}{llll}
0 & 0 & 0 & 1 \\
0 & 0 & 0 & 1 \\
0 & 0 & 0 & 1 \\
0 & 0 & 0 & 1
\end{array}\right)\right| \\
& \Rightarrow m(\varpi)=\left(\begin{array}{cccc}
\varpi & 0 & 0 & -1 \\
0 & \varpi & 0 & -1 \\
0 & 0 & \varpi & -1 \\
0 & 0 & 0 & \varpi-1
\end{array}\right)
\end{aligned}
$$

The following are obtained using Maple (18) software,

$$
\begin{aligned}
& m(\varpi)=\varpi^{3}(\varpi-1) \\
& \Rightarrow \varpi^{3}(\varpi-1)=0 \\
& \Rightarrow \varpi_{1}=1, \varpi_{2}=0, \varpi_{3}=0, \varpi_{4}=0 . \text { Since }\left|\varpi_{i}\right| \leq 1, i=1,2,3,4, \text { the discrete schemes in }(16) \text { is }
\end{aligned}
$$

zero stable. 


\subsection{Convergence}

Since (10), (13) and (16) are both consistent and zero stable, therefore they are convergent.

\subsection{Absolute Stability}

The absolute stability of the regions for the BSM (2) of STDDDEs is plotted for (10), (13) and (16) using first order DDEs of this expression

$$
\begin{aligned}
& d X(z)=\lambda(X(z)+X(z-\tau)) d z+\delta(X(z)+X(z-\tau)) d W(z) \text { for } z>z_{0}, \tau>0 \\
& X(z)=p(z), \text { for } z \leq z_{0}
\end{aligned}
$$

where $p(z)$ is the initial function, $\lambda, \delta$ are complex coefficients, $\tau=c s, c \in Z^{+}, s$ is the step size and $c=\frac{\tau}{s}, c$ is a positive integer. Let $U_{1}=s \lambda$ and $U_{2}=s \delta$, then the $V$-and $R$-stability of (10), (13) and(16) are investigated, plotted and presented in figure 1 to 6 below using of Maple 18 and MATLAB,

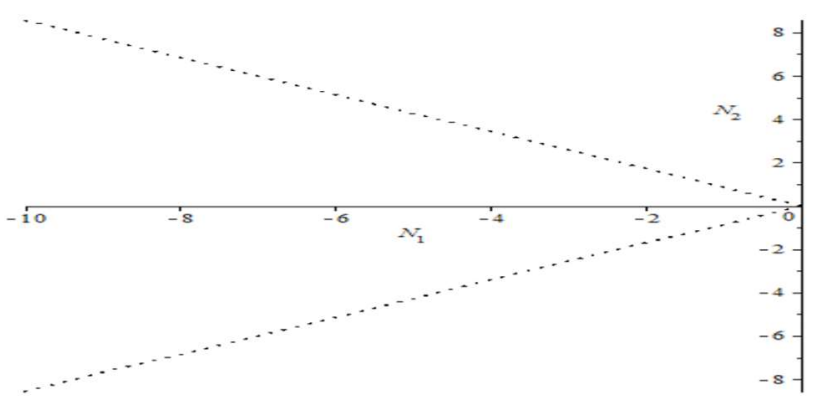

Figure 1: $V$-stability region of (10).

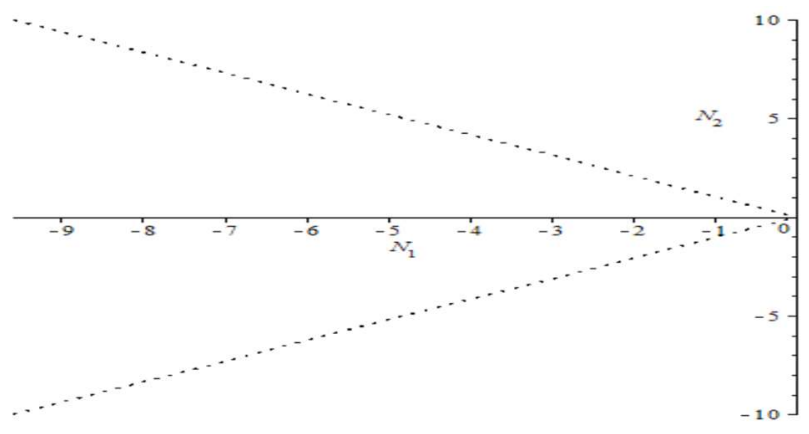

Figure 2: $V$-stability region of (13). 


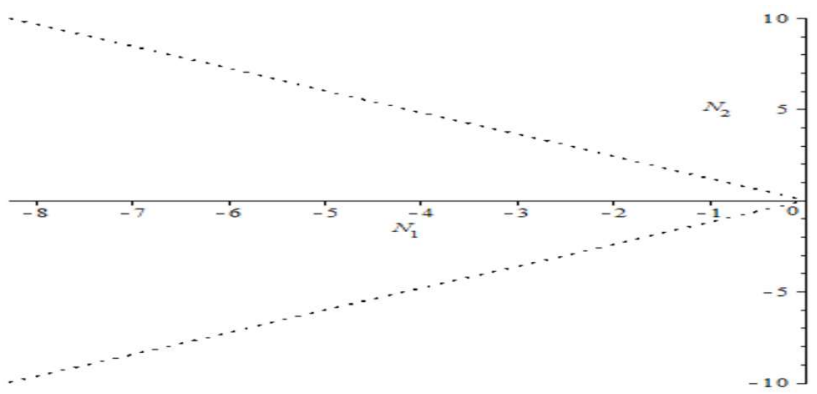

Figure 3: $V$-stability region of (16).

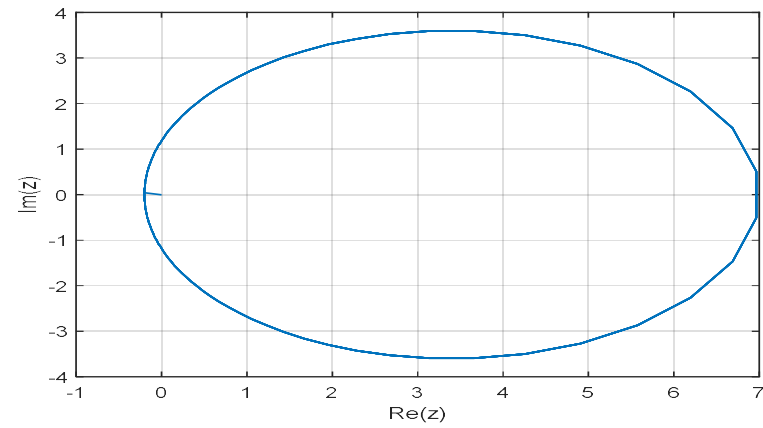

Figure 4: $R$-stability region of (10).

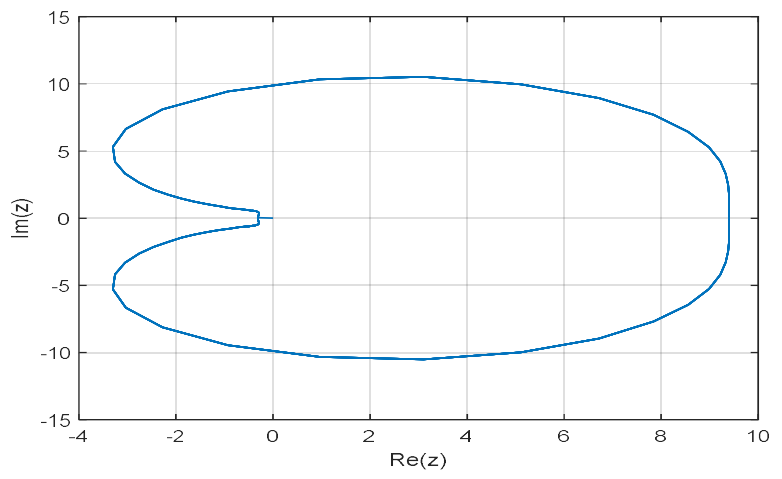

Figure 5: stability region of (13).

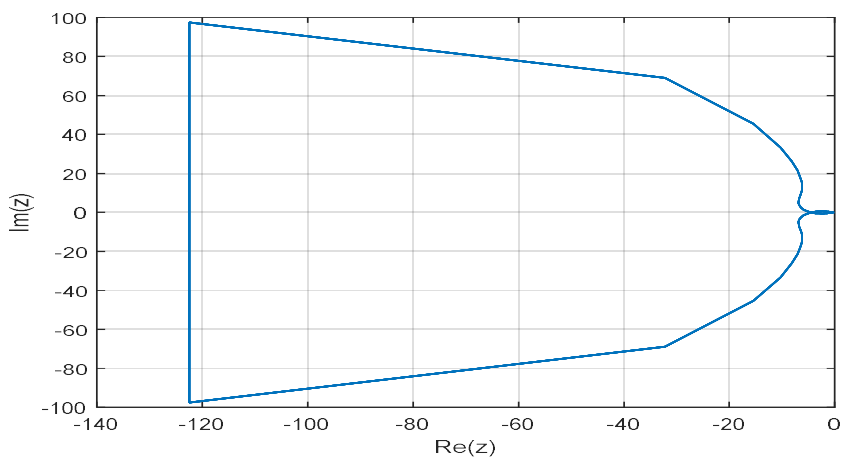

Figure 6: $R$-stability region of (16)The open-ended region shows the $V$-stability regions in Figs 1 to 3 and the enclosed region represents the $R$-stability regions in Figs 4 to 6 . 


\section{NUMERICAL EXPERIMENTS}

The stochastic time-dependent first order delay differential equations shall be experimented using (10), (13) and (16). The delay argument shall be evaluated using the idea of sequence developed by [9] and discrete-time Gaussian white process whose mean $\mu$ is 0 and the standard deviation $\sigma$ is 1derived by [10] to evaluate the wiener term and we inserted them into the stochastic time-dependent first order delay differential equations before obtaining its numerical solutions.

\subsection{Numerical Problems}

\section{Problem 1}

$d X(z)=-1000\left(X(z)+997 e^{-3} X(z-1)+\left(1000-997 e^{-3}\right)\right) d z+\left(X(z)+997 e^{-3} X(z-1)+\left(1000-997 e^{-3}\right)\right) d W(z), 0 \leq z \leq 3$

$p(z)=1+e^{-3 z}, z \leq 0$

Exact solution $p(z)=1+e^{-3 z}$

\section{Problem 2}

$$
\begin{aligned}
& d X(z)=-24\left(X(z)-e^{(-25)} X(z-1)\right) d z+\left(X(z)-e^{(-25)} X(z-1)\right) d W(z), 0 \leq z \leq 3 \\
& p(z)=e^{(-25) z}, z \leq 0
\end{aligned}
$$

Exact solution $p(z)=e^{(-25) z}$

The schemes in (10), (13) and (16) as obtained by the Block Simpson's Methods were applied in solving the problems above and the results obtained were summarized in tables 4.1 to 4.2

Table 4.1: Solution of Problem 1 using the BSM for $k=2,3 \& 4$

\begin{tabular}{|l|l|l|l|l|}
\hline $\mathbf{z}$ & Exact Solution & \multicolumn{1}{|c|}{$\begin{array}{c}\mathbf{k}=\mathbf{2} \\
\text { Approximate } \\
\text { Solution }\end{array}$} & $\begin{array}{c}\text { k=3 } \\
\text { Approximate } \\
\text { Solution }\end{array}$ & $\begin{array}{c}\text { k=4 } \\
\text { Approximate } \\
\text { Solution }\end{array}$ \\
\hline 0.1 & 1.740818221 & 1.982321646 & 1.981311413 & 1.980789406 \\
\hline 0.2 & 1.548811636 & 1.945508631 & 1.948617039 & 1.949472264 \\
\hline 0.3 & 1.40656966 & 1.92414221 & 1.926696137 & 1.923594816 \\
\hline 0.4 & 1.301194212 & 1.89261806 & 1.894266953 & 1.891417142 \\
\hline 0.5 & 1.22313016 & 1.869906585 & 1.869810655 & 1.869661968 \\
\hline 0.6 & 1.165298888 & 1.841944483 & 1.841351787 & 1.843015922 \\
\hline 0.7 & 1.122456428 & 1.819134408 & 1.819109327 & 1.81912834 \\
\hline 0.8 & 1.090717953 & 1.793748162 & 1.79414448 & 1.792862028 \\
\hline 0.9 & 1.067205513 & 1.771486384 & 1.772194252 & 1.771507434 \\
\hline 1 & 1.049787068 & 1.748098559 & 1.748302797 & 1.748367788 \\
\hline 1.1 & 1.036883167 & 1.726705051 & 1.726745883 & 1.726772575 \\
\hline 1.2 & 1.027323722 & 1.70496466 & 1.70472905 & 1.704468768 \\
\hline 1.3 & 1.020241911 & 1.684582015 & 1.684610829 & 1.684629725 \\
\hline 1.4 & 1.014995577 & 1.664264354 & 1.664302563 & 1.664322076 \\
\hline 1.5 & 1.011108997 & 1.644939716 & 1.645123056 & 1.644990337 \\
\hline 1.6 & 1.008229747 & 1.625891249 & 1.625909135 & 1.625641898 \\
\hline 1.7 & 1.006096747 & 1.607621197 & 1.607641115 & 1.607653634 \\
\hline
\end{tabular}




\begin{tabular}{|l|l|l|l|l|}
1.8 & 1.004516581 & 1.589729226 & 1.589658309 & 1.589736402 \\
\hline 1.9 & 1.003345965 & 1.572484278 & 1.5724967 & 1.57251275 \\
\hline 2 & 1.002478752 & 1.555660165 & 1.555660128 & 1.555541399 \\
\hline 2.1 & 1.001836305 & 1.539398152 & 1.539443864 & 1.539415814 \\
\hline 2.2 & 1.001360368 & 1.523568013 & 1.523567277 & 1.523565712 \\
\hline 2.3 & 1.001007785 & 1.508241327 & 1.508247679 & 1.508255676 \\
\hline 2.4 & 1.000746586 & 1.493340866 & 1.493321714 & 1.493286036 \\
\hline 2.5 & 1.000553084 & 1.47890031 & 1.478904144 & 1.478909075 \\
\hline 2.6 & 1.000409735 & 1.464871872 & 1.464870387 & 1.464869226 \\
\hline 2.7 & 1.000303539 & 1.451268738 & 1.451279864 & 1.451275582 \\
\hline 2.8 & 1.000224867 & 1.438059605 & 1.438058619 & 1.438034761 \\
\hline 2.9 & 1.000166586 & 1.425246719 & 1.425248483 & 1.425250867 \\
\hline 3 & 1.00012341 & 1.412808116 & 1.412803216 & 1.412806462 \\
\hline
\end{tabular}

TCPU of STDDDE for $\mathrm{k}=2$ is $0.216 \mathrm{~s}, \mathrm{k}=3$ is $0.123 \mathrm{~s}$ and $\mathrm{k}=4$ is $0.103 \mathrm{~s}$

Table 4.2: Solution of Problem 2 using the BSM for $k=2,3 \& 4$

\begin{tabular}{|c|c|c|c|c|}
\hline $\mathbf{z}$ & Exact Solution & $\begin{array}{c}\mathbf{k}=\mathbf{2} \\
\text { Approximate } \\
\text { Solution }\end{array}$ & $\begin{array}{c}\mathbf{k}=\mathbf{3} \\
\text { Approximate } \\
\text { Solution }\end{array}$ & $\begin{array}{c}\mathrm{k}=4 \\
\text { Approximate } \\
\text { Solution }\end{array}$ \\
\hline 0.1 & 0.082084999 & 0.778687838 & 0.77878502 & 0.778798312 \\
\hline 0.2 & 0.006737947 & 0.606556271 & 0.606527482 & \\
\hline 0.3 & 0.000553084 & 0.472318193 & 0.472347954 & 0.4723648 \\
\hline 0.4 & 4.53999E-05 & 0.367910825 & 0.367857365 & 0.367880599 \\
\hline 0.5 & $3.72665 \mathrm{E}-06$ & 0.286487932 & 0.286491786 & 0.286504799 \\
\hline 0.6 & $3.05902 \mathrm{E}-07$ & 0.223159004 & 0.223112321 & 0.223130535 \\
\hline 0.7 & $2.511 \mathrm{E}-08$ & 0.17377143 & 0.173756393 & 0.173773862 \\
\hline 0.8 & $2.06115 \mathrm{E}-09$ & 0.135358848 & 0.135323535 & 0.135336152 \\
\hline 0.9 & $1.6919 \mathrm{E}-10$ & 0.105402475 & 0.105386391 & 0.105399574 \\
\hline 1 & $1.38879 \mathrm{E}-11$ & 0.082103048 & 0.082073241 & 0.082085411 \\
\hline 1.1 & $1.13999 \mathrm{E}-12$ & 0.063932787 & 0.063919584 & 0.063928047 \\
\hline 1.2 & $9.35762 \mathrm{E}-14$ & 0.049800341 & 0.04977886 & 0.049787558 \\
\hline 1.3 & $7.6812 \mathrm{E}-15$ & 0.038779024 & 0.038766966 & 0.03877447 \\
\hline 1.4 & $6.30512 \mathrm{E}-16$ & 0.030206872 & 0.030192146 & 0.03019764 \\
\hline 1.5 & $5.17556 \mathrm{E}-17$ & 0.023521799 & 0.023512824 & 0.023517897 \\
\hline 1.6 & $4.24835 \mathrm{E}-18$ & 0.018322285 & 0.018311397 & 0.018315884 \\
\hline 1.7 & $3.48726 \mathrm{E}-19$ & 0.014267393 & 0.014261114 & 0.014264382 \\
\hline 1.8 & $2.86252 \mathrm{E}-20$ & 0.011113578 & 0.011106163 & 0.011109131 \\
\hline 1.9 & $2.3497 \mathrm{E}-21$ & 0.008654044 & 0.008649291 & 0.008651782 \\
\hline 2 & $1.92875 \mathrm{E}-22$ & 0.006741067 & 0.006736158 & 0.006738062 \\
\hline 2.1 & $1.58321 \mathrm{E}-23$ & 0.005249212 & 0.005245933 & 0.005247592 \\
\hline 2.2 & $1.29958 \mathrm{E}-24$ & 0.004088875 & 0.004085441 & 0.004086836 \\
\hline 2.3 & $1.06676 \mathrm{E}-25$ & 0.003183974 & 0.003181783 & 0.003182825 \\
\hline 2.4 & $8.75651 \mathrm{E}-27$ & 0.002480159 & 0.002477883 & 0.002478804 \\
\hline 2.5 & $7.18778 \mathrm{E}-28$ & 0.00193128 & 0.001929731 & 0.001930489 \\
\hline 2.6 & $5.90009 \mathrm{E}-29$ & 0.001504373 & 0.001502893 & 0.001503469 \\
\hline 2.7 & 4.84309E-30 & 0.001171444 & 0.00117041 & 0.001170901 \\
\hline 2.8 & $3.97545 \mathrm{E}-31$ & 0.000912498 & 0.000911494 & 0.000911905 \\
\hline 2.9 & $3.26325 \mathrm{E}-32$ & 0.000710556 & 0.00070988 & 0.00071019 \\
\hline 3 & $2.67864 \mathrm{E}-33$ & 0.000553489 & 0.000552834 & 0.000553098 \\
\hline
\end{tabular}

TCPU of STDDDE for $\mathrm{k}=2$ is $0.213 \mathrm{~s}, \mathrm{k}=3$ is $0.121 \mathrm{~s}$ and $\mathrm{k}=4$ is $0.101 \mathrm{~s}$ 


\section{RESULTS AND DISCUSSIONS}

The results obtained after solving some STDDDE using (10), (13) and (16) shall be analyzed to determine the absolute errors and are compared with other existing methods to prove its importance as displayed in the tables and figures below

Table 5.1: Problem 1 Absolute Errors of BSM for $k=2,3$ and 4

\begin{tabular}{|c|c|c|c|}
\hline $\mathbf{z}$ & $k=2$ Error & $\mathbf{k}=3$ Error & $k=4$ Error \\
\hline 0.1 & 0.231503425 & 0.237971185 & 0.241493192 \\
\hline 0.2 & 0.396696995 & 0.399805403 & 0.400660628 \\
\hline 0.3 & 0.51707255 & 0.517125156 & 0.520126477 \\
\hline 0.4 & 0.590123848 & 0.59022293 & 0.593072741 \\
\hline 0.5 & 0.646506425 & 0.646531808 & 0.646580495 \\
\hline 0.6 & 0.676645595 & 0.676651703 & 0.676662899 \\
\hline 0.7 & 0.69667798 & 0.696678012 & 0.696679899 \\
\hline 0.8 & 0.703030209 & 0.703144075 & 0.703426527 \\
\hline 0.9 & 0.704280871 & 0.704301921 & 0.704488739 \\
\hline 1 & 0.698311491 & 0.69858072 & 0.698616729 \\
\hline 1.1 & 0.689821884 & 0.689862408 & 0.689889716 \\
\hline 1.2 & 0.677040938 & 0.677145046 & 0.677405328 \\
\hline 1.3 & 0.664340104 & 0.664387814 & 0.664389918 \\
\hline 1.4 & 0.649268777 & 0.649326499 & 0.649327986 \\
\hline 1.5 & 0.633830719 & 0.63388134 & 0.634014059 \\
\hline 1.6 & 0.617261502 & 0.617412151 & 0.617679388 \\
\hline 1.7 & 0.60152445 & 0.601556887 & 0.601564368 \\
\hline 1.8 & 0.585212645 & 0.585219821 & 0.585241728 \\
\hline 1.9 & 0.569138313 & 0.569166785 & 0.569170735 \\
\hline 2 & 0.553051213 & 0.553062647 & 0.553181376 \\
\hline 2.1 & 0.537560847 & 0.537579509 & 0.537617559 \\
\hline 2.2 & 0.522204645 & 0.522205344 & 0.522206909 \\
\hline 2.3 & 0.507233542 & 0.507247891 & 0.507259894 \\
\hline 2.4 & 0.49253428 & 0.49253945 & 0.492575128 \\
\hline 2.5 & 0.478347226 & 0.478355991 & 0.47836106 \\
\hline 2.6 & 0.464459137 & 0.464460491 & 0.464462652 \\
\hline 2.7 & 0.450965199 & 0.450972043 & 0.450976325 \\
\hline 2.8 & 0.437809738 & 0.437832894 & 0.437833752 \\
\hline 2.9 & 0.425080133 & 0.425084281 & 0.425087897 \\
\hline 3 & 0.412683706 & 0.412684052 & 0.412685306 \\
\hline
\end{tabular}

Table 5.2: Problem 2 Absolute Errors of BSM for $k=2,3$ and 4

\begin{tabular}{|l|l|l|l|}
\hline \multicolumn{1}{|c|}{$\mathbf{z}$} & \multicolumn{1}{|c|}{$\mathbf{k}=\mathbf{2}$ Error } & \multicolumn{1}{|c|}{$\mathbf{k}=\mathbf{3}$ Error } & \multicolumn{1}{c|}{$\mathbf{k = 4}$ Error } \\
\hline 0.1 & 0.69660284 & 0.696700021 & 0.696713313 \\
\hline 0.2 & 0.599818324 & 0.599789535 & 0.599791785 \\
\hline 0.3 & 0.471765109 & 0.47179487 & 0.471811715 \\
\hline 0.4 & 0.367865425 & 0.367811965 & 0.367835199 \\
\hline 0.5 & 0.286484206 & 0.286488059 & 0.286501072 \\
\hline 0.6 & 0.223158698 & 0.223112015 & 0.223160229 \\
\hline 0.7 & 0.173771405 & 0.173756368 & 0.173773837 \\
\hline 0.8 & 0.135338846 & 0.135323532 & 0.13535615 \\
\hline 0.9 & 0.105372475 & 0.10538639 & 0.105409574 \\
\hline
\end{tabular}




\begin{tabular}{|l|l|l|l|}
1 & 0.082003048 & 0.082073241 & 0.082185411 \\
\hline 1.1 & 0.063912787 & 0.063919584 & 0.063928047 \\
\hline 1.2 & 0.049760341 & 0.04977886 & 0.049787558 \\
\hline 1.3 & 0.038759024 & 0.038766966 & 0.03877447 \\
\hline 1.4 & 0.030186872 & 0.030192146 & 0.03019764 \\
\hline 1.5 & 0.023511799 & 0.023512824 & 0.023517897 \\
\hline 1.6 & 0.018310285 & 0.018311397 & 0.018315884 \\
\hline 1.7 & 0.014260393 & 0.01426114 & 0.014264382 \\
\hline 1.8 & 0.011105578 & 0.011106163 & 0.011109131 \\
\hline 1.9 & 0.008634044 & 0.008649291 & 0.008651782 \\
\hline 2 & 0.006734067 & 0.006736158 & 0.006738062 \\
\hline 2.1 & 0.005244212 & 0.005245933 & 0.005247592 \\
\hline 2.2 & 0.004084875 & 0.004085441 & 0.004086836 \\
\hline 2.3 & 0.003180974 & 0.003181783 & 0.003182825 \\
\hline 2.4 & 0.002476159 & 0.002477883 & 0.002478804 \\
\hline 2.5 & 0.00192828 & 0.001929731 & 0.001930489 \\
\hline 2.6 & 0.001502473 & 0.001502893 & 0.001503469 \\
\hline 2.7 & 0.001170044 & 0.00117041 & 0.001170901 \\
\hline 2.8 & 0.000910998 & 0.000911494 & 0.000911905 \\
\hline 2.9 & 0.000708556 & 0.00070988 & 0.000710196 \\
\hline 3 & 0.000552669 & 0.000552834 & 0.000553098 \\
\hline
\end{tabular}

Consider the codes used in the table as stated below

BSA $=$ Block Simpson's Approach for step numbers $k=2,3$ and 4.

CSSEMM $=$ Continuous Split-Step Scheme Euler-Maruyama Approach for step numbers $k=2,3$ and 4 in [6].

$\mathbf{E M M}=$ Euler-Maruyama Approach for step numbers $k=2,3$ and 4 in [7].

HESDBBDFM = Hybrid Extended Second Derivative Backward Differentiation Formulae Methods for step numbers $k=2,3$ and 4 in [11].

ESDBBDFM = Second Derivative Backward Differentiation Formulae Method for step numbers $k=2,3$ and 4 in [14].

MAX = Maximum Error.

Table 5.3: Comparison between the Maximum Absolute Errors of $\operatorname{BSM} k=2,3$ and 4 and $[6,7]$ for fixed step size $s=\mathbf{0 . 0 1}$

\begin{tabular}{|l|l|}
\hline \multicolumn{1}{|c|}{ Approximate Method } & $\begin{array}{c}\text { COMPARED MAXEs } \\
\text { with [6,7] }\end{array}$ \\
\hline $\mathrm{k}=2$ BSM MAX & 0.704280871 \\
\hline $\mathrm{k}=3$ BSM MAX & 0.704301921 \\
\hline $\mathrm{k}=4$ BSM MAX & 0.704488739 \\
\hline $\mathrm{k}=2$ CSSEMM MAX & $4.76 \mathrm{E}-02$ \\
\hline $\mathrm{k}=3$ CSSEMM MAX & $9.17 \mathrm{E}-02$ \\
\hline $\mathrm{k}=4$ CSSEMM MAX & $1.62 \mathrm{E}-01$ \\
\hline
\end{tabular}




\begin{tabular}{|l|l|}
$\mathrm{k}=2$ EMM MAX & $1.84 \mathrm{E}-02$ \\
\hline $\mathrm{k}=3$ EMM MAX & 0.00404 \\
\hline $\mathrm{k}=4$ EMM MAX & 0.000973 \\
\hline
\end{tabular}

Table 5.4: Comparison between the Maximum Absolute Errors of BSM $k=2,3$ and 4 and $[6,7]$ for fixed step size

$$
s=\mathbf{0 . 0 1}
$$

\begin{tabular}{|l|l|}
\hline \multicolumn{1}{|c|}{ Approximate Method } & \multicolumn{1}{c|}{$\begin{array}{c}\text { COMPARED } \\
\text { MAXEs with [6,7] }\end{array}$} \\
\hline $\mathrm{k}=2$ BSM MAX & 0.69660284 \\
\hline $\mathrm{k}=3$ BSM MAX & 0.696700021 \\
\hline $\mathrm{k}=4$ BSM MAX & 0.696713313 \\
\hline $\mathrm{k}=2$ CSSEMM MAX & $3.18 \mathrm{E}-02$ \\
\hline $\mathrm{k}=3$ CSSEMM MAX & $5.90 \mathrm{E}-02$ \\
\hline $\mathrm{k}=4$ CSSEMM MAX & $1.37 \mathrm{E}-01$ \\
\hline $\mathrm{k}=2$ EMM MAX & $1.09 \mathrm{E}-01$ \\
\hline $\mathrm{k}=3$ EMM MAX & 0.04912833 \\
\hline $\mathrm{k}=4$ EMM MAX & 0.02437045 \\
\hline
\end{tabular}

Table 5.5: Comparison between the Maximum Absolute Errors of BSM $k=2,3$ and 4 and $[11,14]$ for fixed step size $s=\mathbf{0 . 0 1}$

\begin{tabular}{|l|l|}
\hline \multicolumn{1}{|c|}{ Numerical Method } & \multicolumn{1}{c|}{$\begin{array}{c}\text { COMPARED MAXEs } \\
\text { with }[11,14]\end{array}$} \\
\hline $\mathrm{k}=2$ BSM MAX & 0.704280871 \\
\hline $\mathrm{k}=3$ BSM MAX & 0.704301921 \\
\hline $\mathrm{k}=4$ BSM MAX & 0.704488739 \\
\hline $\mathrm{k}=2$ HESDBBDFM MAX & 0.695948088 \\
\hline $\mathrm{k}=3$ HESDBBDFM MAX & 0.695129625 \\
\hline $\mathrm{k}=4$ HESDBBDFM MAX & 0.695893683 \\
\hline $\mathrm{k}=2$ ESDBBDFM MAX & $5.04 \mathrm{E}-02$ \\
\hline $\mathrm{k}=3$ ESDBBDFM MAX & $6.69 \mathrm{E}-02$ \\
\hline $\mathrm{k}=4$ ESDBBDFM MAX & $7.09 \mathrm{E}-02$ \\
\hline
\end{tabular}

Table 5.6: Comparison between the Maximum Absolute Errors of BSM $k=2,3$ and 4 and $[11,14]$ for fixed step size $s=0.01$

\begin{tabular}{|l|l|}
\hline Approximate Method & $\begin{array}{l}\text { COMPARED MAXEs } \\
\text { with }[11,14]\end{array}$ \\
\hline $\mathrm{k}=2$ BSM MAX & 0.69660284 \\
\hline $\mathrm{k}=3$ BSM MAX & 0.696700021 \\
\hline $\mathrm{k}=4$ BSM MAX & 0.696713313 \\
\hline $\mathrm{k}=2$ HESDBBDFM MAX & 0.696784203 \\
\hline $\mathrm{k}=3$ HESDBBDFM MAX & 0.696773595 \\
\hline
\end{tabular}




\begin{tabular}{|l|l|}
$\mathrm{k}=4$ HESDBBDFM MAX & 0.696766115 \\
\hline $\mathrm{k}=2$ ESDBBDFM MAX & $2.08 \mathrm{E}-03$ \\
\hline $\mathrm{k}=3$ ESDBBDFM MAX & $1.91 \mathrm{E}-03$ \\
\hline $\mathrm{k}=4$ ESDBBDFM MAX & $3.43 \mathrm{E}-03$ \\
\hline
\end{tabular}

The compared Maximum Error for BSM with $[6,7]$ and $[11,14]$ are shown in Figs.7 to 10 as follows,

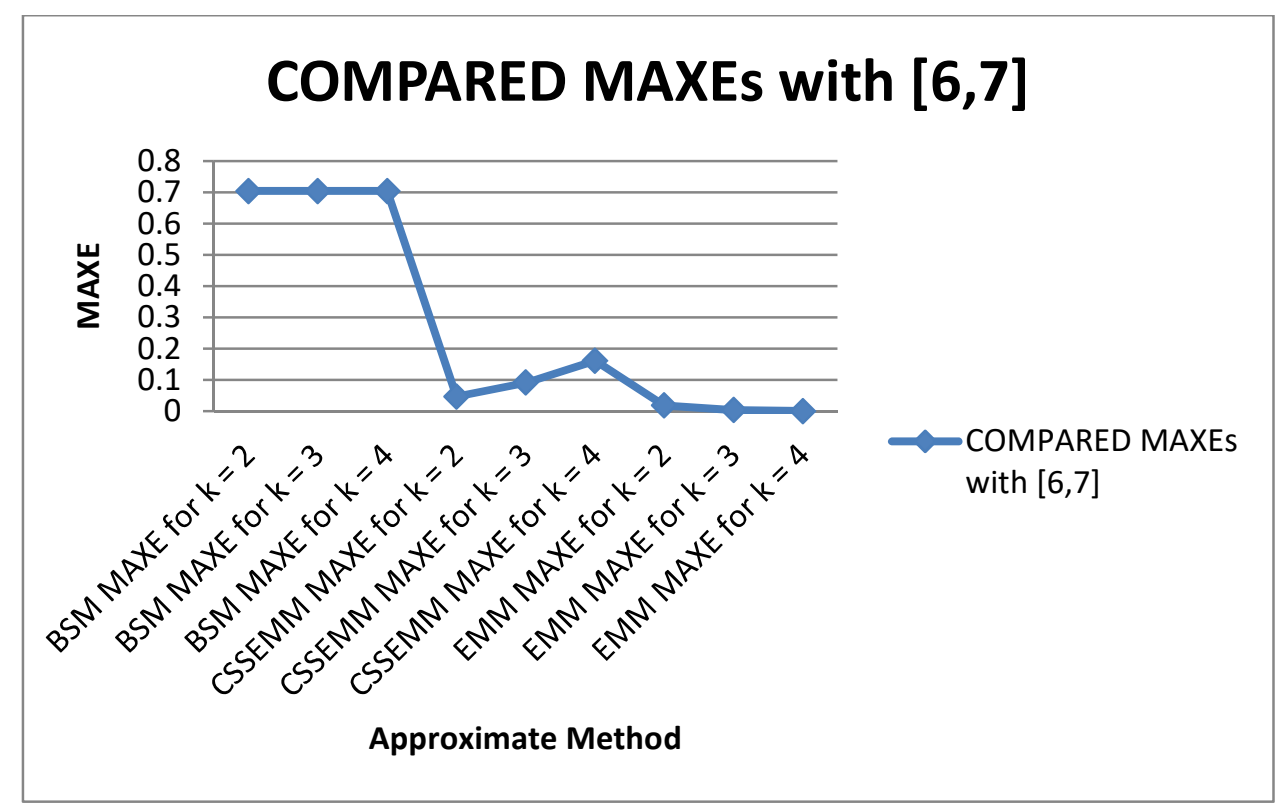

Figure 7: Compared MAX for BSM with [6, 7].

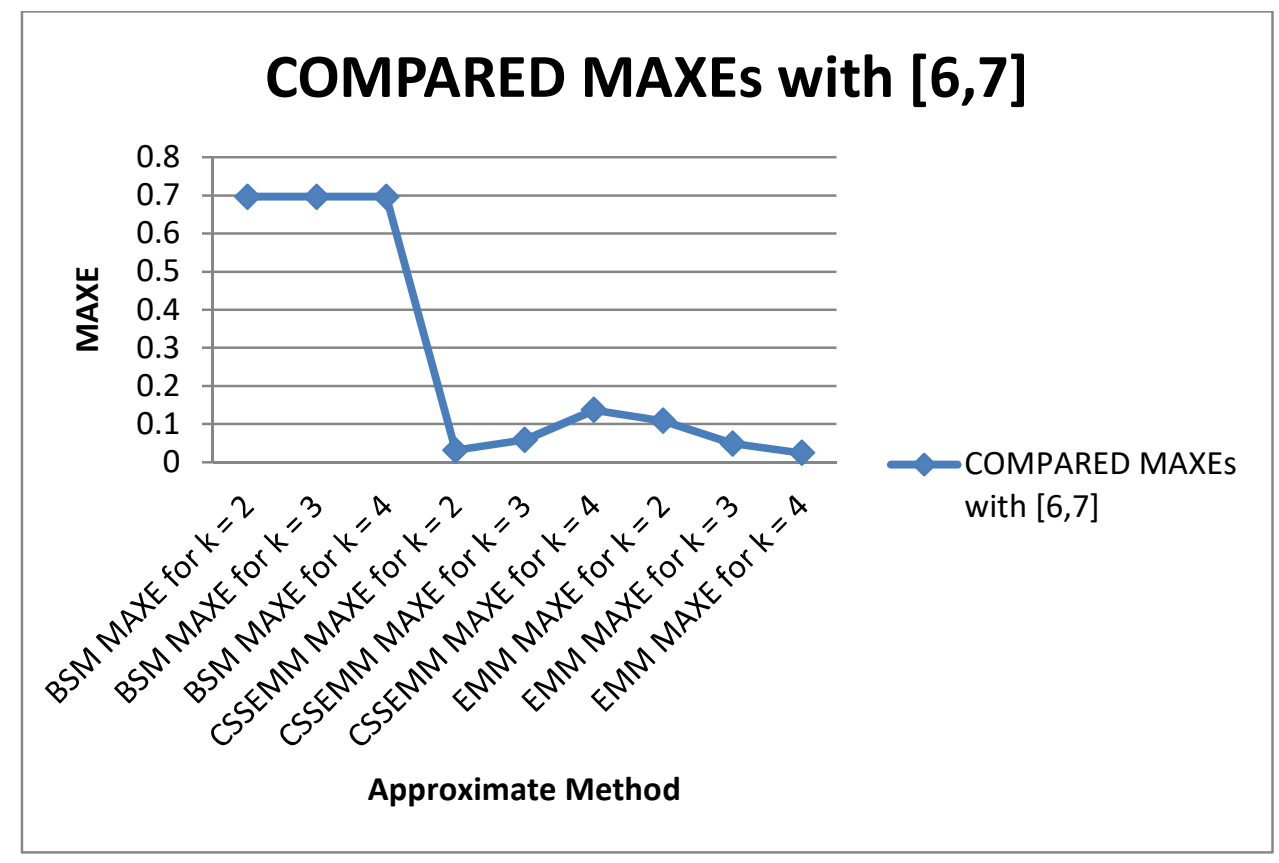

Figure 8: Compared MAX for BSM with [6, 7]. 


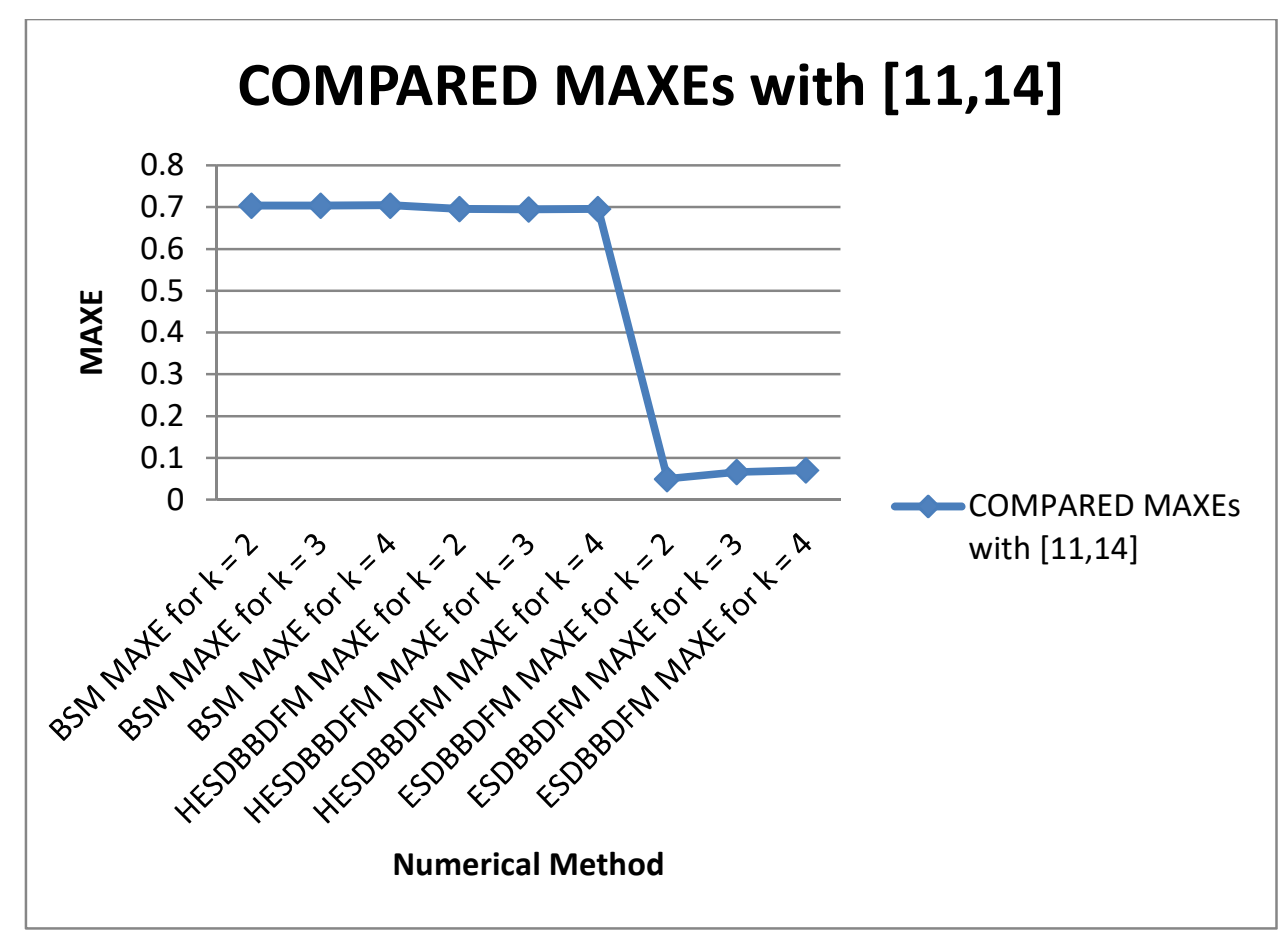

Figure 9: Compared MAX for BSM with [11, 14].

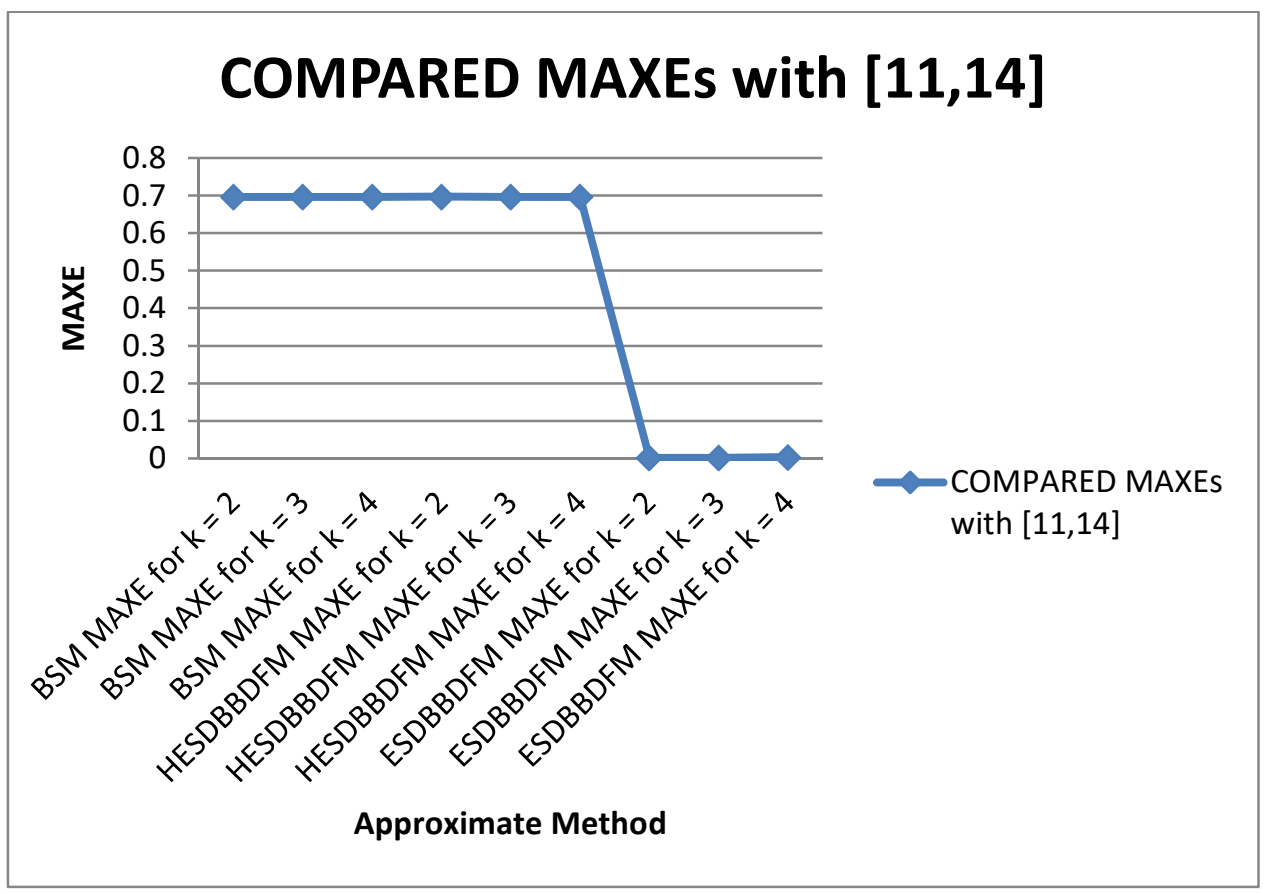

Figure 10: Compared MAX for BSM with [11, 14].

\subsection{CONCLUSIONS}

In conclusion, the discrete schemes of (10), (13) and (16), were obtained from the respective continuous formulation of each step number $k=2,3$, and 4 and were proved to be convergent, $V$ - and $R$-stable. Also, it was observed in tables 5.1 to 5.2 that the higher step number $k=4$ of BSM performed better and faster than the lower step numbers of $k=3$ and 2 after comparing them with the exact solutions. Comparing BSM with other existing methods, it proved its importance over 
other existing methods in terms of accuracy at fixed pence size $S$ as showed in table 5.3 to 5.6 and figures 7 to 10 . Therefore, it is recommended that BSM schemes for pace numbers $k=2,3$, and 4 are suitable for solving STDDDEs. Further research should be carried-out for step numbers $k=5,6,7 \ldots$ on the derivation of discrete schemes of BSM for approximate solutions of STDDDEs without the introduction of intercalation methods in evaluating the lag term.

\section{REFERENCES}

1. Akhtari, B., Babolian, E., Neuenkirch, A. (2015) An Euler scheme for stochastic delay differential equations on unbounded domains: pathwise convergence. Discrete Contin. Dyn. Syst., Ser.B 20(1), 23-38

2. Wang, X., Gan, S. (2011). The improved split-step backward Euler method for stochastic differential delay equations. Int. J. Comput. Math. 88 (11), 2359-2378

3. Kazmerchuk, Y.I. (2005) Pricing of derivatives insecurity markets with delayed response. Ph.D. thesis, University of York, Toronto, Canada 14.

4. Kazmerchuk, Y.I., Wu, J.H. Stochastic state-dependent delay differential equations with applications in finance. Funct. Differ. Equ.11(1-2), 77-86

5. Zhang, H., Gan, S., Hu, L. (2009). The split-step backward Euler method for linear stochastic delay differential equations. Comput. Appl. Math.225(2),558-568

6. Evelyn, B. (2000). Introduction to the numerical analysis of stochastic delay differential equations. Journal of Computational and Applied Mathematics. 125,297-307.

7. Bahar, A. (2019). Numerical solution of stochastic state-dependent delay differential equations: convergence and stability. Advances in Deference Equations, A Springer Open Journal.396 https://doi.org/10.1186/s13662-019-2323-x

8. Majid, Z.A., Radzi, H.M.,\& Ismail, F. (2013).Solving delay differential equations by the five-point one-step block method using Neville's interpolation. International Journal of Computer Mathematics. http://dx.doi.org/10.1080/00207160.2012. 754015.

9. Sirisena, U. W., \& Yakubu S. Y. (2019). Solving delay differential equation using reformulated backward differentiation methods. Journal of Advances in Mathematics and Computer Science, 32(2), 1 - 15.

10. Robert, G., (2006). Course materials for 6.450 principles of digital communications I. MIT Open Course Ware (http://ocw.mit.edu/), Massachusetts Institute of Technology.

11. C. Chibuisi, B. O. Osu, C. Olunkwa, S. A. Ihedioha, and S. Amaraihu (2020). Computational treatment of first order delay differential equations using hybrid extended second derivative block backward differentiation formulae. EJ-MATH, European Journal of Mathematics and Statistics DOI: http://dx.doi.org/10.24018/ejmath.2020.1.1.8. 1(1),1-14.

12. Osu, B.O., Chibuisi, C., Okwuchukwu, N.N., Olunkwa, C., Okore, N.A. (2020),Implementation of third derivative block backward differentiation formulae for solving first order delay differential equations without interpolation techniques. Asian journal of Mathematics and Computer Research (AJOMCOR) 27(4),1-26.

13. Osu, B.O., Chibuisi, C., Edeki, S.O., Okwuchukwu, N.N. and Olunkwa, C.(2020).

14. Numerical solutions of first order delay differential equations using second derivative block backward differentiation formulae. To be present in the International Journal of Mathematical Models and Methods in Applied Sciences of North Atlantic University Union (NAUN). 
15. Chibuisi, C., Osu, B.O., Ihedioha, S. A., Olunkwa, C., Okwuchukwu, N.N., Okore, N.A. (2020). The construction of extended second derivative block backward differentiation formulae for numerical solutions of first order delay differential equations. Journal of Multidisciplinary Engineering Science Studies (JMESS) 6(12), 3620-3631.

16. J. D., Lambert "Computational methods in ordinary differential equations”, New York, USA. John Willey and Sons Inc.(1973).

17. Bellen A, Zennaro M. Numerical methods for delay differential equations. New York: Oxford University Press; 2003.

18. Onumanyi P, Awoyemi DO, Jator SN, Sirisena UW. New linear multistep methods with continuous coefficients for first order initial value problems. Journal of Nigerian Mathematical Society. 1994;13: 37-51.

19. Trong Trung Nguyen, Van Tu Duong, Huy Hung Nguyen, Thanh Phuong Nguyen \& Viet Thang Tran, "Remote Discrete-Time Model Reference Adaptive Control Of A Two-Wheeled Mobile Robot “, International Journal of Mechanical and Production Engineering Research and Development (IJMPERD), Vol. 9, Issue 4, pp. 1325-1334

20. Anshu Mala Kispotta, "PID Control Strategy in Networked Control System ", International Journal of Computer Science and Engineering (IJCSE), Vol. 3, Issue 3, pp. 67-82

21. Pankaj S. Ardak \& Atul B. Borade, "An EPQ Model with Varying Rate of Deterioration and Mixed Demand Pattern ", International Journal of Mechanical and Production Engineering Research and Development (IJMPERD), Vol. 7, Issue 6, pp. 11-20 